\title{
Clinicopathological Features of Ductal Carcinoma In Situ from ${ }^{18}$ F-FDG-PET Findings
}

\author{
TAKAAKI FUJII, KEIKO YANAI, SHOKO TOKUDA, YUKO NAKAZAWA, SASAGU KUROZUMI, \\ SAYAKA OBAYASHI, REINA YAJIMA, TOMOKO HIRAKATA and HIROYUKI KUWANO \\ Division of Breast and Endocrine Surgery, Department of General Surgical Science, \\ Graduate School of Medicine, Gunma University, Gunma, Japan
}

\begin{abstract}
Aim: The presence of ductal carcinoma in situ (DCIS) can increase the risk of developing an invasive ductal carcinoma (IDC), but it is difficult to predict what will occur if a DCIS is left untreated. We reported the usefulness of ${ }^{18} \mathrm{~F}$-fluorodeoxyglucose positron emission tomography (FDG-PET) for DCIS, and that the presence of FDG uptake in the tumor could be considered a predictor of invasive potential in patients with DCIS. In this study, we retrospectively evaluated the clinicopathological features of DCIS by using FDG-PET findings, and we evaluated the possibility of using FDG-PET in DCIS cases as a biomarker of which lesions will go on to become invasive. Patients and Methods: We investigated the cases of 185 consecutive patients with primary breast cancer who were diagnosed as having DCIS or IDC and underwent FDG-PET preoperatively. Results: We divided the cases into two groups on the basis of histology; DCIS vs. IDC $(n=171)$. The DCIS cases were divided into two groups on the basis of FDG uptake in the primary tumor. Fourteen of the 185 patients $(7.4 \%)$ were revealed to have a DCIS. The analysis revealed that the $S U V_{\text {max }}$ and the number of cases not detected by FDG-PET were significantly different between the DICS and IDC groups. The extent of the primary tumor was not significantly different between the two groups. In six cases (42.9\%) of the 14 DCIS cases, no FDG uptake was detected by FDG-PET. The extent of tumor did not significantly differ between the two groups. In addition, all six cases without FDG uptake were of the diffuse-spread type, without mass formation. All eight cases with mass formation had FDG uptake. Conclusion: Our present findings suggest that the
\end{abstract}

Correspondence to: Takaaki Fujii, MD, Ph.D., FACS, Department of General Surgical Science, Graduate School of Medicine, Gunma University, 3-39-22 Showa-machi, Maebashi, Gunma 371-8511, Japan. Tel: +81 0272208224, Fax: +81 0272208230, e-mail: ftakaaki@gunma-u.ac.jp

Key Words: FDG-PET, DCIS, breast cancer, ductal spread.
FDG-PET uptake reflects tumor burden or tumor density, which should be considered to be associated with the presence of invasion.

Ductal carcinoma in situ (DCIS) is a pre-cancer or noninvasive cancer of the breast. DCIS does not spread beyond the duct into any of the surrounding tissue in the breast. The presence of DCIS could increase the risk of developing an invasive ductal carcinoma (IDC), but it is difficult to predict what will occur if a DCIS is left untreated. There is no biomarker that can be used to predict which lesions will go on to become invasive or instead remain stable 'noninvasive' disease.

In recent years, clinical applications of ${ }^{18} \mathrm{~F}$ fluorodeoxyglucose-positron emission tomography (FDGPET) have undergone explosive growth for diagnosing the stage or recurrence in breast cancer; however, the diagnostic utility of FDG-PET for breast cancer is controversial (1-11). Several research groups have conducted that the use of FDGPET for the detection of primary breast cancer is currently not advised, mainly because of its low sensitivity in cases of smaller carcinoma or histology, including lobular carcinoma or DCIS (1-4). The majority of FDG-PET studies have been carried out on patients with invasive breast cancer because DCIS has been reported to be poorly imaged by FDG-PET $(5,6)$. FDG-PET measures glucose metabolism, which reflects the biological aggressiveness of cancers $(1,2,10$ 14). Thus, FDG-PET may provide biological information about the tumor growth potential. In fact, several studies have reported that high FDG uptake is predictive of poor prognosis and aggressive features in patients with breast cancer $(1,2,10-14)$.

There exist only few reports regarding the role of FDGPET in the detection of DCIS of the breast (15-17), and the standardized uptake value (SUV) pattern of DCIS of the breast on FDG-PET examination is not fully understood. The findings of our previous study, demonstrated (1) the usefulness of FDG-PET for DCIS and (2) that the presence of FDG uptake in the tumor could be considered a predictor 
for invasive potential in cases with DCIS cases (17). In the present study, we evaluated the clinicopathological features of DCIS obtained using FDG-PET findings, and we considered the possibility for additional usefulness of FDGPET in DCIS cases as a biomarker to predict which lesions will go on to become invasive.

\section{Patients and Methods}

We retrospectively investigated the cases of 185 consecutive patients with primary breast cancer who were diagnosed as having DCIS or IDC and underwent FDG-PET preoperatively at the Gunma University (Gunma, Japan), in the period from January 2010 to October 2015. All patients had undergone radical breast surgery.

We excluded patients with incomplete clinical information, those who underwent preoperative chemotherapy, and male patients. Among the 185 patients, 14 (7.6\%) were diagnosed as having DCIS. The patients underwent FDG-PET/computed tomography (CT) as part of the routine standard of care, without deviating from the main protocol. The maximum standardized uptake value $\left(\mathrm{SUV}_{\max }\right)$ of each primary tumor was calculated in a routine clinical fashion. Written consent was obtained from all patients for the use of their records and imaging in future studies.

The details extracted from the database were age, histological type, the size of the invasive primary tumor, the size of extent of tumor, the presence of lymphatic or vascular invasion, the nuclear grade, the estrogen receptor (ER) expression and progesterone receptor $(\mathrm{PgR})$ expression status, the human epidermal growth factor receptor 2 (HER2) score of the primary tumor, the axillary lymph node status, the $\mathrm{SUV}_{\max }$ of the primary tumor, and the visibility of the detected lesion by FDG-PET. The ER and PgR expressions were assessed by ALLRED scores, with an ALLRED score of $\geq 3$ defined as indicating ER and PgR positivity $(18,19)$.

Statistical analysis. We divided the breast cancer cases into two groups on the basis of histology, i.e. DCIS versus IDC. We divided the DCIS cases into two groups on the basis of FDG uptake in the primary tumor. We conducted a univariate statistical analysis using Fisher's exact test or the $\chi^{2}$ test with Yates' correction. Student's $t$ test was used to compare the two groups. Differences were considered significant when $p<0.05$. To test the independence factors related to negative FDG uptake, we entered the variables with a likelihood of $p<0.05$ into were entered into a multivariate logistic regression model.

\section{Results}

Presence of FDG uptake was not associated with tumor extension in patients with IDC and DCIS. A total of 185 cases were included in the analysis. In 14 patients $(7.4 \%)$, the histopathological examination revealed DCIS. We divided the 185 breast cancer cases into two groups based on the histology of the primary tumor, i.e., DCIS $(n=14)$ and IDC $(n=171)$. Table I summarizes the patients' characteristics and the results of the univariate analysis conducted to determine differences in clinicopathologic variables between DCIS and IDC. The univariate analysis revealed that the $\mathrm{SUV}_{\text {max }}$ and the number of cases not detected by FDG-PET were significantly different between the DICS and IDC groups. The extent of primary tumor was not significantly different between the two groups.

FDG uptake in the DCIS patients was associated with mass formation. In six (42.9\%) of the 14 DCIS cases, no FDG uptake was detected by FDG-PET. We divided the 14 DCIS cases into two groups based on the presence of FDG uptake in the primary tumor. Table II summarizes both the patients' characteristics and the results of the univariate analysis conducted to determine the relationships between negative FDG uptake in the primary tumor and various clinicopathologic variables. The univariate analysis revealed that age was significantly associated with negative FDG uptake in the primary tumor; however, the multivariate analysis revealed that age was not a significant factor, and the extent of the tumor did not differ significantly between the two groups. In addition, all six of the cases without FDG uptake were the diffuse-spread type, without mass formation, whereas all eight of the cases with mass formation had FDG uptake.

\section{Discussion}

FDG-PET has high specificity but mediocre sensitivity for identifying primary breast cancer, and it can differentiate breast cancers from benign lesions with $66 \%-96 \%$ sensitivity and $83-100 \%$ specificity $(3,4,7)$. In our previous study, the overall sensitivity for the detection of all breast cancers was $88.6 \%$ and the false-negative rate of the FDG-PET evaluation of primary breast cancer was $11.4 \%$ (3). Many studies have evaluated factors associated with the FDG avidity of the primary tumor in breast cancer and the tumor size or histological type of breast cancer has been reported to be associated with a greater likelihood of FDG uptake (3).

Several studies have reported that the $\mathrm{SUV}_{\max }$ correlates with the size of a tumor to a certain value, according to the resolution of the PET scanner, known as the partial volume effect (20). However, our present analysis revealed that the extent of the tumor did not significantly differ between the DCIS and IDC groups. The FDG uptake may be determined mainly by the number of viable tumor cells $(21,22)$, but not by ductal spread. Thus, the FDG-PET evaluation was not sufficient for evaluating the ductal spread of a tumor. However, the $\mathrm{SUV}_{\max }$ of patients with a nodular growth pattern is significantly higher than the $S_{U V} V_{\text {max }}$ of patients with a diffuse growth pattern $(13,22,23)$. In the present study, all cases with a nodular growth pattern had FDG uptake in the tumors, and all of the cases without FDG uptake had a diffuse growth pattern. The FDG-PET findings reflected the tumor burden or tumor density, which should be considered to be associated with the presence of invasion.

FDG-PET measures glucose metabolism, which reflects biological information about the tumor growth potential. 
Table I. Patients characteristics and clinicopathological features associated with invasion.

\begin{tabular}{lccc}
\hline & DCIS $(\mathrm{n}=14)$ & IDC $(\mathrm{n}=171)$ & $p$-Value \\
\hline Age (years) & $60.6 \pm 12.6$ & $59.2 \pm 12.2$ & 0.697 \\
CEA (ng/ml) & $1.67 \pm 0.92$ & $2.52 \pm 2.46$ & 0.238 \\
CRP (mg/dl) & $0.08 \pm 0.13$ & $0.12 \pm 0.52$ & 0.749 \\
SUV ${ }_{\text {max }}$ & $1.49 \pm 2.10$ & $3.26 \pm 2.79$ & 0.022 \\
not detected by FDG-PET & 6 & 17 & 0.002 \\
Extention of tumor (mm) & $38.8 \pm 29.6$ & $30.9 \pm 12.3$ & 0.165 \\
Node metastases (n) & 0 & 44 & 0.065 \\
ER (n) & 14 & 147 & 0.276 \\
PgR (n) & 11 & 136 & 0.796 \\
HER2 (n) & 2 & 28 & 0.862 \\
Nuclear grade 3 (n) & 2 & 62 & 0.171 \\
ly (n) & 0 & 97 & $<0.001$ \\
v (n) & 0 & 43 & 0.070 \\
\hline
\end{tabular}

Values are expressed as mean \pm SD. IDC, Invasive ductal carcinoma DCIS, ductal carcinoma in situ; CRP, C-reactive protein; CEA, carcinoembryonic antigen.

DCIS patients have shown a lower level of FDG uptake, and DCISs are detected at a significantly lower sensitivity than IDCs. In the present study, the $\mathrm{SUV}_{\max }$ of the primary tumors and the presence of FDG uptake in the tumors were associated with the presence of invasion. Infiltrating ductal carcinomas have a higher level of FDG uptake and are therefore detected with significantly higher sensitivity compared to DCISs.

In our previous study, the presence of FDG uptake in the tumor could be considered as a predictor for invasion in DCIS cases confirmed by needle biopsy. That finding, combined with our present results, suggests that FDG uptake reflects not only the biological aggressiveness of tumors but also the tumor cell density of intraductal carcinoma, which may reflect tumor invasion or future invasion, and that FDGPET is thus useful for the prediction of the invasion of DCIS. This possibility should be investigated in further studies.

This study has several potential limitations. The major limitation is the retrospective method of data collection. The number of cases was also relatively small. However, the clinical implications of the data we obtained are very important. There have been only a few reports about the role of FDG-PET in DCIS because the majority of prior FDGPET studies were carried out on IDC patients. To the best of our knowledge, this is the first report describing the usefulness of FDG-PET as a biological predictor of invasion in patients with DCIS. Additional research is needed to explore the significance of FDG uptake for predicting invasion in patients diagnosed with DCIS.
Table II. Patients characteristics and clinicopathological features associated with FDG uptake in DCIS.

\begin{tabular}{lccc}
\hline & \multicolumn{2}{c}{ FDG uptake } & \\
\cline { 2 - 3 } & Absent (n=6) & Present (n=8) & $p$-Value \\
\hline Age (years) & $51.0 \pm 10.7$ & $67.8 \pm 8.6$ & 0.011 \\
CEA (ng/ml) & $0.97 \pm 0.43$ & $2.34 \pm 0.62$ & \\
CRP (mg/dl) & $0.10 \pm 0.16$ & $0.05 \pm 0.05$ & 0.574 \\
Extention of tumor (mm) & $40.3 \pm 24.0$ & $37.6 \pm 33.1$ & 0.878 \\
ER (n) & 6 & 8 & - \\
PgR (n) & 5 & 6 & 0.615 \\
HER2 (n) & 1 & 1 & 0.692 \\
Grade (n) & & & 0.896 \\
low & 3 & 5 & \\
intermediate & 2 & 2 & \\
high & 1 & 1 & \\
\hline
\end{tabular}

Values are expressed as mean $\pm \mathrm{SD}$. IDC, Invasive ductal carcinoma; DCIS, ductal carcinoma in situ; CRP, C-reactive protein; CEA, carcinoembryonic antigen.

In conclusion, our present findings suggest that the results of an FDG-PET evaluation reflect tumor burden or tumor density. In patients with DCIS, the presence of FDG uptake in the tumor may be considered a predictor of future invasion.

\section{Acknowledgements}

The Authors would like to thank Saitoh Y, Kanai H, Yano T, Matsui Y, Takada F and Okada A for their secretarial assistance. Supported by Grants-in-Aid from the Japanese Ministry of Education, Culture, Sports, Science and Technology (T.F.).

\section{References}

1 Rostom AY, Powe J, Kandil A, Ezzat A, Bakheet S, el-Khwsky F, el-Hussainy G, Sorbris R and Sjoklint O: Positron emission tomography in breast cancer: a clinicopathological correlation of results. Br J Radiol 72: 1064-1068, 1999.

2 Avil N, Rose CA, Schelling M, Dose J, Kuhn W, Bense S, Weber W, Ziegler S, Graeff $\mathrm{H}$ and Schwaiger M: Breast imaging with positron emission tomography and fluorine-18 fluorodeoxyglucose: use and limitations. J Clin Oncol 18: 34953502,2000

3 Fujii T, Yajima R, Tsuboi M, Higuchi T, Obayashi S, Tokiniwa $\mathrm{H}$, Nagaoka $\mathrm{R}$, Takata $\mathrm{D}$, Horiguchi $\mathrm{J}$ and Kuwano $\mathrm{H}$ : Clinicopathological features of cases with primary breast cancer not identified by ${ }^{18}$ F-FDG-PET. Anticancer Res 36: 3019-3022, 2016.

4 Fujii T, Yajima R, Kurozumi S, Higuchi T, Obayashi S, Tokiniwa H, Nagaoka R, Takata D, Horiguchi J and Kuwano H: Clinical significance of $18 \mathrm{~F}-\mathrm{FDG}-\mathrm{PET}$ in invasive lobular carcinoma. Anticancer Res 36: 5481-5485, 2016. 
5 Quon A and Gambhir SS: FDG-PET and beyond: molecular breast cancer imaging. J Clin Oncol 23: 1664-1673, 2005.

6 Vincenzi B, Calvieri A, Santini D, Altomare V and Tonini G: Occasional FDG-PET recognition of in situ breast cancer. Ann Oncol 18: 1584-1585, 2007.

7 Koolen BB, van der Leij F, Vogel WV, Rutgers EJ, Vrancken Peeters MJ, Elkhuizen PH and Valdés Olmos RA: Accuracy of ${ }^{18}$ F-FDG PET/CT for primary tumor visualization and staging in T1 breast cancer. Acta Oncol 53: 50-57, 2014.

8 Groves AM, Shastry M, Ben-Haim S, Kayani I, Malhotra A, Davidson T, Kelleher T, Whittaker D, Meagher M, Holloway B, Warren RM, Ell PJ and Keshtgar M: Defining the role of PETCT in staging early breast cancer. Oncologist 17: 613-619, 2012.

9 Hodgson NC and Gulenchyn KY: Is there a role for positron emission tomography in breast cancer staging? J Clin Oncol 26: 712-720, 2008.

10 Fujii T, Yajima R, Tatsuki H, Oosone $\mathrm{K}$ and Kuwano $\mathrm{H}$ : Implication of ${ }^{18} \mathrm{~F}$-Fluoroxyglucose uptake of affected axillary lymph nodes in cases with breast cancer. Anticancer Res 36: 393-397, 2016.

11 Fujii T, Yajima R, Tatsuki H, and Kuwano H: Prediction of extracapsular invasion at metastatic sentinel nodes and nonsentinel lymph nodal metastases by FDG-PET in cases with breast cancer. Anticancer Res 36: 1785-1789, 2016.

12 Aogi K, Kadoya T, Sugawara Y, Kiyoto S, Shigematsu H, Masumoto $\mathrm{N}$ and Okada M: Utility of $18 \mathrm{~F}$ FDG-PET/CT for predicting prognosis of luminal-type breast cancer. Breast Cancer Res Treat 150: 209-217, 2015.

13 Ohara M, Shigematsu H, Tsutani Y, Emi A, Masumoto N, Ozaki $\mathrm{S}$, Kadoya T and Okada M: Role of FDG-PET/CT in evaluating surgical outcomes of operable breast cancer - usefulnesss for malignant grade of triple-negative breast cancer. Breast 22: 958963, 2013

14 Sanli Y, Kuyumcu S, Ozkan ZG, Isik G, Karanlik H, Guzelbey B, Turkmen C, Ozel S, Yavuz E and Mudun A: Increased FDG uptake in breast cancer is associated with prognostic factors. Ann Nucl Med 26: 345-350, 2012.

15 Azuma A, Tozaki M, Ito K, Fukuma E, Tanaka T and O'uchi T: Ductal carcinoma in situ: correlation between FDG-PET/CT and histopathology. Radiat Med 26: 488-493, 2008.
16 Shigematsu H, Kadoya T, Masumoto N, Matsuura K, Emi A, Kajitani K, Amioka A and Okada M: Role of FDG-PET/CT in prediction of underestimation of invasive breast cancer in cases of ductal carcinoma in situ diagnosed at needle biopsy. Clin Breast Cancer 14: 358-364, 2014.

17 Fujii T, Yajima R, Tatsuki H, Oosone K and Kuwano H: ${ }^{18} \mathrm{~F}-$ fluorodeoxyglucose uptake as predictor for invasion in preoperatively diagnosed breast ductal carcinoma in situ: significance in cases without mass formation. Mol Clin Oncol, 2017. doi: 10.3892/mco.2017.1304 [Epub ahead of print]

18 Allred DC, Harvey JM, Berardo M and Clark GM: Prognostic and predictive factors in breast cancer by immunohistochemical analysis. Mod Pathol 11: 155-168, 1998.

19 Shousha S: Oestrogen receptor status of breast carcinoma: Allred/H score conversion table. Histopathology 53: 346-347, 2008.

20 Hoffman EJ, Huang SC and Phelps ME: Quantitation in positron emission computed tomography: Effect of object size. J Comput Assist Tomogr 3: 299-308, 1979.

21 Higashi K, Anaira CC and Wahl RL: Does FDG uptake measure proliferative activity of human cancer cells? In vitro comparison with DNA flow cytometory and tritiated thymidine uptake. J Nucl Med 34: 414-419, 1993.

22 Avril N, Menzel M, Dose J, Schellin M, Weber W, Janicke F, Nathrath W and Schwaiger M: Glucose metabolism of breast cancer assessed by ${ }^{18} \mathrm{~F}-\mathrm{FDG}$ PET: histologic and immunohistochemical tissue analysis. J Nucl Med 42: 9-16, 2001.

23 Owaki T, Kijima Y, Yoshinaka H, Uenososno Y, Yoshioka T, Natsugoe $\mathrm{S}$ and Aikou T: Ductal carcinoma in situ of the breast detected by F-18 Fluorodeoxyglucose positron emission tomography. Breast Cancer 13: 210-213, 2006. 